\title{
Philosophical Consideration of Sustainable Development in Research Areas
}

\author{
Meiqing Lin ${ }^{1, a}$, Weiming Huang ${ }^{2, b}$ and Yong $\mathrm{Zhu}^{3}$ \\ ${ }^{1}$ Department of Information Countermeasure, Air Force Early Warning College, Wuhan,China \\ ${ }^{2}$ Nanning audit center, Audit commission of the central military commission,Nanning,China \\ ${ }^{3}$ Department of Scientific Research, Air Force Early Warning College, Wuhan, China

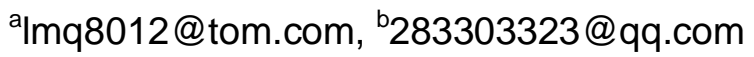

Keywords: Innovation; Scientific research culture; Cognitive model; Sustainable development.

\begin{abstract}
According to the problem of thinking way and sustainable development in research areas, on account of historical world outlook and systems ideas, and methodology of scientific research work which based on "connection and development" is established. Then, thinking and methods of marketing management in electricity supplier field are used for references, and some rational thinking is done about key problems during research and study, thus key elements including resource integration, propaganda campaign and so on are induced and summarized, which can provide a reference for scientific research management department and scientific research staff.
\end{abstract}

\section{Introduction}

Scientific research is an initiative and creative work. During this working, emerging realities should be based, by seeking effective solution and approach, technical progress and technological innovation is realized. Scientific research is also unusually arduous task. In the base of having specific research goal, exploration must be done in the ocean of knowledge. So, doing innovation by appropriate cognitive model and method, which is much necessary for increasing efficiency and standards of scientific research.

Scientific research area is import position of development and innovation. Development potential and power of one unit or institution lie on its innovation ability and level to a large extent in scientific research area. Thus, innovation and development in scientific research area is a worth pondering problem for research group and scientific research institution and so on. In simple terms, sustainable development in scientific research area is an idea which can take aim at certain demand areas, and based on a certain technological front, and depend on some resource and management guarantee, then, sustainable innovation and rolling development in a field of science and technology is realized.

At present, some research and exploration is carried on in scientific and technological innovation field. In literature [1] a system innovation thought is provided, which is according to sustainable innovation problem of enterprise. In literature [2], innovation and development in market field is discussed. In literature [4], some creative thinking for demand management issues during project management is done. In literature [5], quality control problem for management of research and development is debated. Research above on is good reference and experience for the work of this paper.

According to the innovation driven development strategy, the $13^{\text {th }}$ five-year scientific research and development planning demonstration is launching, and seminar on scientific and technological innovation is needed. In this paper, cognitive model of technological innovation, methodology of scientific effort and key elements of sustainable development are going to be researched, which is focus on scientific research culture, and provide a reference for scientific research and scientific research team construction. 


\section{Vertical and Horizontal Two Meta Cognitive Model of Technological Innovation}

In our regular life, there are usually two kinds of thinking model including vertical and horizontal. The former is focus on correlation and comparison between entities from a spatial perspective. For example, sometime we try to extract commonalities in different fields. The latter is focus on exploration to evolution and development, and to predict the developing trend, which is from the angle of time history. Then, we can combine the above two models into Two-Meta cognitive model, that is vertical-meta and horizontal-meta.

- vertical-meta

Vertical-meta is cognitive object centered, and a cognitive space is opened up, then similar objects in this cognitive space are used into this cognitive framework. By comparison and analysis between the object cognitive object and the other cognitive units in this cognitive framework, we can get the state, insufficient and development direction of the object. For example, according to one's self-consciousness, it is wise to compare oneself with around others, then get an overall grasp of personal skill, making and level and so on, thus know one's short board and short, which is the base to establish the direction of development.

- horizontal-meta

Horizontal-meta is to start from the cognitive object itself, and examine continuously the cognitive object by a timeline, and search the insufficient of the object, and extract law to forecast prospect, thus direction is clear. For example, it is viable to recognize a person that is by above thinking model, and to review the past and analysis the status, thus get the overall of the future.

The above two meta can be combine with each other, and the list is arranged in random order, which is instructive for self-cognition, need recognition and so on.

\section{Scientific research work methodology connection and development based}

Based on the above cognitive model, with the world outlook of historical materialism and system thought, when we carry out various work and conduct problem analysis, two points should be watched out, one is expansibility, the other is relevance. Just as we often say, we should have the insight into the connection and development of everything. With scientific research going on, we can build the work methodology connection and development based. The methodology has two dimensions, which are connection dimension and development dimension. As shown in Figure 1.

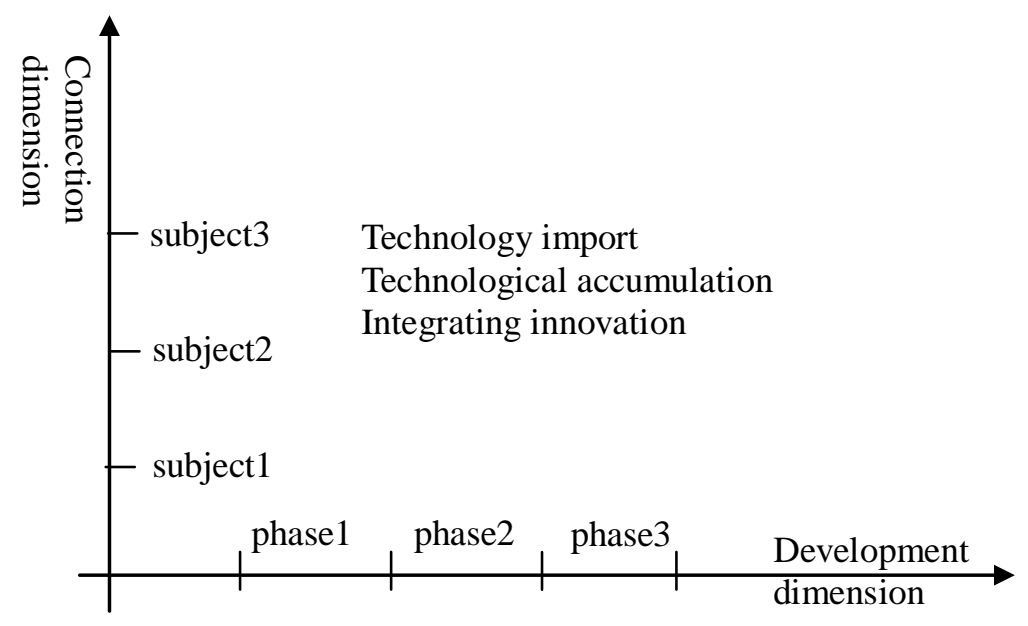

Fig.1. two dimensional analysis of scientific research

- connection dimension

For connection dimension, we should pay attention to using some thinking such as lateral contrast, drawing lessons from, import and so on. Thus, during the scientific research work going on, we should take related subjects and areas in view, and attach importance to the universality and universality of some thinking, method and means in several subject fields. It provides evidences from another side for necessary and important of disciplinary crossing and integration. Perhaps, the problems which can not be break through in this field, are break though by the aid of some thinking and means in related fields. 


\section{- development dimension}

Development dimension of scientific research work is necessarily much accounted of. It is necessary that we can gain a full understanding of disciplinary bases by protracting and backward extension from this subject. We can even recur to the thinking and method as regression and prediction, and dig deeper into superiority, insufficient and development trends of subjects. Then we can foothold the resource and superiority, and grasp several points of disciplinary growth, and promote innovation and development of the scientific research work. It is by standing upon the shoulders of Giants.

\section{Sustainable development of scientific area}

In recent years, internet, e-marketing and other fields is developing rapidly, many concepts as disruptive technologies, technical warning and so on have attracted more, some useful strategies and methods of market management are respected. These strategies and methods have some reference value for sustainable development of scientific area. Combining the practice of scientific research in recent years, some scientific research work factors can be summarized, as shown in Figure2. This can be a reference for scientific innovation and construction and development of research group.

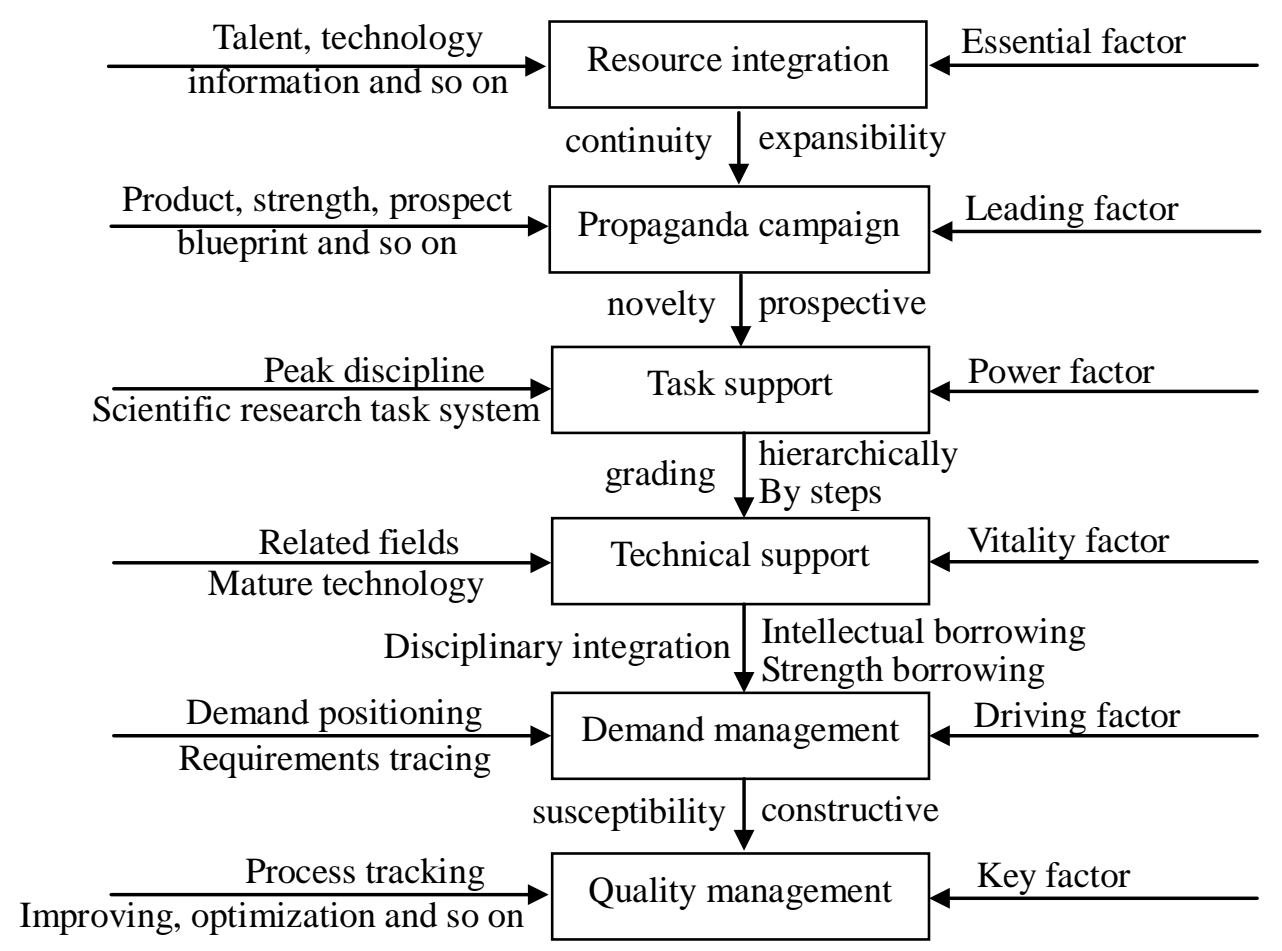

- resource integration

Fig.2. key factors of scientific research work

Resource integration includes brainpower integration, technology resources integration and information resources integration. By integrating the talents, technology means and platform, demand and technology information and so on, a solid foundation is laid for scientific research work carrying on. It can be said that resource integration is essential factor for sustainable development of scientific area. Much more, resource integration is sustainable and repeatable, which refract the construction and development process of group from the point of view of research group.

- propaganda campaign

Propaganda content includes product, strength, prospect and so on, which provide support for opening the opportunity window of further development. It can be said that propaganda campaign is leading factor for sustainable development of scientific area. Much more, propaganda is a kind innovation in itself, which can promote the strategy development of a group or academy, and guarantee the motivation and vitality of scientific effort, and is also the important way for building team spirit, developing institutional culture and establishing core values.

task support

Task support is around the main line of strategic development of scientific research, to build research effort 
system, and to carry on, improve and optimize by stages, by levels and by steps. It can be said that task support is power factor for sustainable development of scientific area. Much more, the mission development of process is really a process of scientific research practice, which is an important growing point of innovation. During the process, it is very important to plan the scientific research task and carry out long-team planning.

- technical support

Technical support is to use external force scientifically and neatly during carrying on scientific research work, and to improve the overall strength of scientific research by means of intellectual borrowing such as tracking technology front and using mature technology and so on. It can be said that technical support is vitality factor for sustainable development of scientific area, Much more, technological process is also long-term and dynamic, then technical progress and development in related field should be followed. During the process, cross-disciplinary training mode and discipline integration thought are very important.

- demand management

Demand management mainly includes demand positioning, requirements tracing, demand forecast and so on, which is to combine certain scientific requirements of innovation and development in a certain context of strategic environment and orientation, and to capture relevant requirements, and to manage continually, then to provide the springhead for carrying on scientific research work. It can be said that demand management is driving factor for sustainable development of scientific area. During the process, it is worth paying attention to carry forward the standardization and engineered of demand management.

- quality management

Quality management is an important mean and way to ensure the valid match between scientific research output and user satisfaction, which is important context of refined scientific research management. It can be said that quality management is key factor for sustainable. At present, quality management in scientific area is paid more and more attention to, but there is certain distance between scientific product quality improvement and ensure goal attainment. How to realize the mutual promotion and jointly promotion between scientific quality management and innovation? It perhaps is key problem of innovation and development in scientific area in future.

\section{Conclusion}

In summary, according to a specific scientific research problem, we can refer to the above train of thought, and firstly examine the discipline foundation, problem, superiority, orientation and so on from longitudinal direction, and focus on research needs, and determine basic methods and technological frame. Then based on that, lateral thinking should be done, and take adjacent discipline and area in view, by having full reference to effective thinking and method of adjacent area, we can enrich the subject methods and technical system. Finally, by using connection thinking with development thinking, solution and technological approaches of target problem are established.

Above thought and methods combine connection dimension and development dimension, which not only can avoid the duplication of similar projects, but also take full advantage of latest ideas and achievements of natural science, and break down discipline barrier, and fuzzy discipline boundary, which is useful to get quickly solutions of scientific problems, and then raise innovation efficiency and level of scientific research.

Combined with the above analysis, some advises are provided for innovation and development of scientific research work area, as follows:

- Having prospect of discipline and technology development, we should regard long term planning and design of scientific research work

- Aiming at strategic line of scientific development, scientific research task and frame should be constructed.

- Regarding disciplinary crossing and integration, innovation and development of scientific research can be promoted continually.

- By the aid of latest technical means and management ideology, scientific research demand management can be strengthened.

- Combined scientific research innovation demand, quality management should be regarded

\section{References}

[1] Mingfeng Qiu. System Innovation Path of Enterprise Sustainable Development [J]. Journal of Neijiang Vocational and Technical College, 2015 9(01):51-53.

[2] Jinlong Pang, Yufeng Liu, Congwei Lin. A Brief Analysis of new ways of Marketing Development. Telecom World [J]. 2015 (04):203-204.

[3] Haizhou Liu, Jian Ye, Wengman Wu. Resource Integration and Innovation Advantage—Development 
Opportunities of Operators in Mobile Internet Era. Information and Communication Technology [J]., 2015 (04): 52-57.

[4] Rui Ding. Analysis of Requirement Management in Software Project [J]. Operating Management, 2012 (03):61-62.

[5] Li Yang. Research on the Application and Development of Total Quality Management in Scientific Research Management [J]. Management Observer, 2015 19(07):29-30. 